\title{
Preparation and Characterization and Biodistribution Studies of Lomustine Loaded PLGA Nanoparticles by Interfacial Deposition Method
}

Archana Mehrotra ${ }^{1 *}$ and Jayanta Kumar Pandit ${ }^{2 *}$

${ }^{1}$ Rakshpal Bahadur College of Pharmacy, Bukhara More, Bareilly, Uttar Pradesh, India

${ }^{2}$ Department of Pharmaceutics, Institute of Technology, Banaras Hindu University, Varanasi, Uttar Pradesh, India

\begin{abstract}
The incorporation of lomustine, a hydrophobic anticancer drug into PLGA nanoparticles by interfacial deposition method was optimized. Based on the optimal parameters, it was found that lomustine-PLGA nanoparticles with acceptable properties could be obtained. Optimization of formulation variables to control the size and drug entrapment efficiency of the prepared nanoparticles seems to be based on the same scientific principles as drug-loaded nanoparticles prepared by nanoprecipitation, solvent evaporation method. The process was the most important factor to control the particle size, while both the drug-polymer interaction and the partition of drug in organic and aqueous phases were the crucial factors to govern the drug entrapment efficiency. PLGA concentration at lower level $(100 \mathrm{mg}), 1: 5$ organic phase: aqueous ratio, 1\%w/v PVA concentration, 3\%w/v pluronic F68 achieved smaller particle size. Additionally, L:G ratio of PLGA $75: 25$, lower volume of organic solvent (1:10 organic phase: aqueous phase), higher initial drug content $(10 \mathrm{mg})$ enhanced the drug entrapment efficiency and maintained lomustine concentration in blood for an extended time period, elevated lomustine concentration in lungs and slowed the elimination of lomustine. The biodistribution profiles of prepared nanoparticles in albino mice showed higher plasma drug concentration for longer period of time, elevated drug concentration in lungs and slow elimination from kidney. No toxic effects of prepared nanoparticles were observed in histopathological examination of lungs and kidney. The systematic investigation reported here promises the development of PLGA nanoparticles loaded with lomustine when tested in Lung Cancer cell line L132 and toxicological/ histopathological studies in albino mice.
\end{abstract}

Keywords: Lomustine; PLGA Nanoparticles; Interfacial deposition; Pluronic F68; PVA

\section{Introduction}

One of the most widely used polymers for nanoparticles is the biodegradable and biocompatible poly (d,l-lactide-co-glycolide) (PLGA), which has been approved by the FDA for certain human clinical uses. Poly (lactide-co-glycolide) polymers undergo hydrolysis upon administration to the body, forming biologically compatible and metabolizable moieties (lactic acid (L) and glycolic acid (G)) that are eventually removed by the citric acid cycle [1].

The unique structure of PLGA nanoparticles, composed of a hydrophilic surface and a hydrophobic core, provides a drug-carrying reservoir and also enables them to dissolve in aqueous solutions. Many approaches are proposed for the preparation of PLGA nanoparticles. The emulsification-evaporation method, spontaneous emulsificationsolvent diffusion method (SESD) [2], and nanoprecipitation method are all widely used for preparing various diameters of PLGA nanoparticles. During nanoparticles formation by emulsification evaporation and SESD approaches, toxic organic solvents such as $\mathrm{CH}_{2} \mathrm{Cl}_{2}$ and $\mathrm{CHCl}_{3}$ are usually used. To meet the requirement for clinical use, the residual solvents should be completely removed from the PLGA particles [3].

Interfacial deposition is a process used for the production of nanocapsules; however, this is not a polymerization technique but an emulsification/solidification technique. In interfacial deposition, a fifth compound is introduced, of oil nature, miscible with the solvent of the polymer but immiscible with the mixture. The polymer deposits on the interface between the finely dispersed internal phase droplets and the aqueous phase, forming nanocapsules. An aqueous solution is used as the dispersing medium. The main difference is that polymers such as PLA are dissolved together with the drug in a solvent mixture (eg, benzyl benzoate, acetone, and phospholipids). This mixture is injected slowly into a stirred aqueous medium, resulting in the deposition of the polymer in the form of nanoparticles of about $230 \mathrm{~nm}$ in size [4].
The interfacial deposition method is recommended for the incorporation of hydrophobic drugs into polymeric nanoparticles $[5,6]$. As described by several authors and also as demonstrated in the work of Fonseca et al. [7] the establishment of a protocol that allows nanoparticles precipitation, while avoiding extensive diffusion of the drug along with the solvent aiming at obtaining high values of drug encapsulation is a challenging issue [8]. Also, the aggregation of PLGA NPs during solvent-evaporation process is a notable problem regardless different preparation method. In order to prevent PLGA nanoparticle aggregation, polymer stabilizers are often used. Many stabilizers such as poly (vinyl alcohol) (PVA), poly(vinyl pyrrolidone) (PVP), Tween 80, Fluonic 127 (poloxamer 407), Fluonic 68 (poloxamer 188), didodecyl dimethyl ammonium bromide (DMAB) are also excellent stabilizer candidates [3]. These stabilizers are coated on the surface of PLGA nanoparticles and can affect the zeta potential, particle size and particle surface properties. PLGA nanospheres have been suggested to be a good carrier because of the safety and achieving sustained release. The degradation time of PLGA can be altered from weeks to months by varying the molecular weight of the copolymer or the lactic acid to glycolic acid ratio in copolymer [9].

*Corresponding authors: Archana Mehrotra, Rakshpal Bahadur College of Pharmacy, Bukhara More, Budaun Road, Near Doordarshan Kendra/ITBP, Bareilly, Uttar Pradesh India, 243001, Tel: +919319318909; E-mail: archana.mehrotra@rediffmail.com

Jayanta Kumar Pandit, Department of Pharmaceutics, IT, Banaras Hindu University, Varanasi-221005, Uttar Pradesh, India, Tel: +919451570863; E-mail: jkpandit@bhu.ac.in

Received August 24, 2015; Accepted September 16, 2015; Published December 01,2015

Citation: Mehrotra A, Pandit JK (2015) Preparation and Characterization and Biodistribution Studies of Lomustine Loaded PLGA Nanoparticles by Interfacial Deposition Method. J Nanomed Nanotechnol 6: 328. doi:10.4172/2157-7439.1000328

Copyright: (c) 2015 Mehrotra A, et al. This is an open-access article distributed under the terms of the Creative Commons Attribution License, which permits unrestricted use, distribution, and reproduction in any medium, provided the original author and source are credited. 
Citation: Mehrotra A, Pandit JK (2015) Preparation and Characterization and Biodistribution Studies of Lomustine Loaded PLGA Nanoparticles by Interfacial Deposition Method. J Nanomed Nanotechnol 6: 328. doi:10.4172/2157-7439.1000328

The objective of this work was to obtain an optimum formulation of PLGA nanoparticles by utilizing factors which are likely to influence their mean size, surface morphology, encapsulation efficiency, and drug release. Considering lomustine as a lipophilic model drug, biodegradable lomustine loaded nanoparticles were prepared by interfacial deposition method. This is based on the interfacial deposition of a polymer following displacement of a semipolar solvent miscible with water from a lipophilic solution. The solubility and compatibility of the drug was tested with materials used for preparing the drug delivery system. The influence of various formulation components such as PLGA concentration, grade of PLGA: PURASORB PDLG 5002 and PDLG 7502, organic phase volume, lomustine content, PVA and Pluronic F68 concentrations as $1 \%$ and 3\%w/v, PVA and pluronic F68 solution content (aqueous phase volume) 1:10 as that of organic solution on the characteristics of nanoparticles was investigated. The cytotoxicity of the selected PLGA-lomustine nanoparticles was evaluated on the L132 Human Lung cancer cells. Also stability and biodistribution of selected nanoparticles was observed with toxicological/ histopathological studies.

\section{Materials and Methods}

\section{Materials}

Polymer PLGA was a gift sample from Purac Biomaterials, Gorinchem, The Netherlands. Lomustine pharmaceutical grade was obtained from Fujian Provincial Medicines and Health Products, Xiamen Import and Export Corporation (China). Acetone used of Qualigens, India; Pluronic F68 was used of Hi-Media, India. All other chemicals were of analytical grade.

\section{Methods}

\section{Preformulation studies}

Saturation solubility of lomustine in PVA solution, Pluronic F68 solution, in polymers PDLG 5002, PDLG 7502 and in phosphate buffer saline $\mathbf{p H}$ 7.4: Lomustine solubility in oversaturation conditions $(\mathrm{C}>10 \times \mathrm{Cs})$ was obtained in PVA solution $(1 \% \mathrm{w} / \mathrm{v})$, PluronicF68 solution $(1 \% \mathrm{w} / \mathrm{v})$, PURASORB PDLG5002 solution $(1 \% \mathrm{w} / \mathrm{v})$, PURASORB PDLG7502 solution (1\%w/v), Phosphate buffer saline (PBS) pH 7.4 by dispersing $300 \mathrm{mg}$ of drug in $100 \mathrm{ml}$ of respective solutions for water solvent and $3 \mathrm{~g}$ or more until saturation of drug in $5 \mathrm{ml}$ of respective solutions for acetone solvent. The suspensions were stirred under constant magnetic stirring at $37 \pm 0.5^{\circ} \mathrm{C}$ for $24 \mathrm{~h}$ (adequate time for equilibration), filtered through a syringe filter $(0.45$ $\mu \mathrm{m})$ and then assayed spectrophotometrically at $230 \mathrm{~nm}$ by dilution with ethanol $95 \% \mathrm{v} / \mathrm{v}$ for acetone solvent, and at $230.4 \mathrm{~nm}$ by dilution with PBS pH 7.4 for water solvent.

Differential Scanning Calorimetry (DSC) of lomustine, 1:1 mixtures of drug:polymer (PDLG 5002 and PDLG 7502) : The DSC runs were carried out on the DSC (DSC-60, Shimadzu Co., Kyoto, Japan) instrument. Five to ten milligrams of drug and polymer was placed in aluminium pans and hermatically sealed. The heating rate was $10^{\circ} \mathrm{C} / \mathrm{min}$; nitrogen served as purge gas and the system was cooled by liquid nitrogen.

Fourier Transform Infrared Spectroscopy (FTIR) of pure drug, polymer and 1:1 mixture of drug, polymer (PDLG 5002 and PDLG7502): Infrared spectra were recorded on Shimadzu 8400S FT-IR spectrophotometer using $\mathrm{KBr}$ pellet method.

In-vitro lomustine release: The in vitro drug release of lomustine nanoparticles was determined on a Franz diffusion cell. As regards to sink condition, $2 \mathrm{mg}$ of lomustine in $2 \mathrm{ml}$ PBS pH 7.4 was placed in donor site and $50 \mathrm{ml}$ PBS in receptor chamber with membrane (cellulose membrane, mw cut off $12,000 \mathrm{Da}$ ) placed in between, incubated at $37^{\circ} \mathrm{C}$ under magnetic stirring $(200 \mathrm{rpm})$. At specific time intervals, $1 \mathrm{ml}$ of medium was removed and replaced with the same volume of fresh PBS. The samples were analyzed spectrophotometrically at $230.4 \mathrm{~nm}$.

\section{Preparation of nanoparticles}

Nanoparticles were prepared by the modified interfacial deposition method [7]. Components are listed in Table 1. An organic solution was prepared by dispersing PLGA and lomustine in $10 \mathrm{ml}$ acetone one by one. The organic phase was added drop wise into $1 \% \mathrm{w} / \mathrm{v}$ polyvinyl alcohol (PVA) aqueous solution 1:5 under magnetic stirring at room temperature. Stirring was continued until complete evaporation of organic solvent. Subsequently, nanoparticles were separated by centrifugation in Remi C-24 cooling centrifuge at $19,000 \mathrm{rpm}$ at $4^{\circ} \mathrm{C}$ for $40 \mathrm{~min}$. The drug-loaded nanoparticles (DNPs) were diluted with $10 \mathrm{ml}$ of distilled water, the dispersion was centrifuged and supernatant was discarded. This was repeated three times to remove traces of acetone from the DNPs. The final volume was adjusted to $10 \mathrm{ml}$ with water. The residue was used for further characterization by evaporating to dryness to constant weight at reduced pressure at $35^{\circ} \mathrm{C}$. Blank nanoparticles were prepared as per above method omitting the drug.

For study of effect of different formulation components, the following variables were studied. a) PLGA concentration, b) PURASORB PDLG 5002 and PDLG 7502, c) Organic phase volume, d) Lomustine content, e) PVA and Pluronic F68 concentrations as $1 \%$ and $3 \% \mathrm{w} / \mathrm{v}, \mathrm{f}$ ) PVA and Pluronic F68 solution content (aqueous phase volume) 1:10 as that of organic solution.

\section{Nanoparticle characterization}

Particle size, polydispersity index (PDI), zeta potential and scanning electron microscopy (SEM): The method used by Mehrotra and Pandit [10] was used. The Zetasizer used was of Malvern instruments, UK DTS Ver 5.10 and SEM was performed on Hitachi Japan S-3400N.

Drug content and encapsulation efficiency: The method published by Mehrotra and Pandit [10] was used.

\begin{tabular}{|c|c|c|c|c|c|}
\hline $\begin{array}{l}\text { S. } \\
\text { No. }\end{array}$ & $\begin{array}{l}\text { Batch } \\
\text { code }\end{array}$ & PLGA (mg) & $\begin{array}{c}\text { Acetone } \\
(\mathrm{ml})\end{array}$ & $\begin{array}{l}\text { Lomustine } \\
\text { (mg) }\end{array}$ & Stabilizer solution (ml) \\
\hline 1 & PD1 & 100 mg PDLG5002 & 10 & 1 & $1: 5,1 \% w / v$ PVA \\
\hline 2 & PD2 & 200 mg PDLG5002 & 10 & 1 & $1: 5,1 \% w / v$ PVA \\
\hline 3 & PD3 & 300 mg PDLG5002 & 10 & 1 & $1: 5,1 \% w / v$ PVA \\
\hline 4 & PD4 & 100 mg PDLG7502 & 10 & 1 & $1: 5,1 \% w / v$ PVA \\
\hline 5 & PD5 & 200 mg PDLG7502 & 10 & 1 & $1: 5,1 \% w / v$ PVA \\
\hline 6 & PD6 & 300 mg PDLG7502 & 10 & 1 & $1: 5,1 \% w / v$ PVA \\
\hline 7 & PD7 & 100 mg PDLG5002 & 20 & 1 & $1: 5,1 \% w / v$ PVA \\
\hline 8 & PD8 & 100 mg PDLG5002 & 30 & 1 & $1: 5,1 \% w / v$ PVA \\
\hline 9 & PD9 & 100 mg PDLG5002 & 10 & 5 & $1: 5,1 \% w / v$ PVA \\
\hline 10 & PD10 & 100 mg PDLG5002 & 10 & 10 & $1: 5,1 \% w / v$ PVA \\
\hline 11 & PD11 & 100 mg PDLG5002 & 10 & 1 & $1: 5,3 \% w / v$ PVA \\
\hline 12 & PD12 & 100 mg PDLG5002 & 10 & 1 & 1:5, 1\%w/v Pluronic F68 \\
\hline 13 & PD13 & 100 mg PDLG5002 & 10 & 1 & 1:5, 3\%w/v Pluronic F68 \\
\hline 14 & PD14 & 100 mg PDLG5002 & 10 & 1 & $1: 10,1 \% w / v$ PVA \\
\hline 15 & PD15 & 100 mg PDLG5002 & 10 & 1 & $1: 10,1 \%$ w/v Pluronic F68 \\
\hline
\end{tabular}

L/G ratio of: For PDLG 5002 is 50:50; For PDLG 7502 is $75: 25$

Table 1: Components of various batches of fabricated lomustine nanoparticles. 
Citation: Mehrotra A, Pandit JK (2015) Preparation and Characterization and Biodistribution Studies of Lomustine Loaded PLGA Nanoparticles by Interfacial Deposition Method. J Nanomed Nanotechnol 6: 328. doi:10.4172/2157-7439.1000328

Page 3 of 13

\section{In- vitro drug release study}

The method published by Mehrotra and Pandit [10] was used. Here all method is similar only lomustine was determined by dialysis method using Franz diffusion cell. Lomustine nanoparticles in $2 \mathrm{ml}$ PBS pH 7.4 was placed in donor site and $50 \mathrm{ml}$ PBS in receptor chamber with membrane (cellulose membrane, mw cut off 12,000 Da) placed in between which was washed with double distilled water previously and incubated at $37^{\circ} \mathrm{C}$. For evaluation of release kinetics, the obtained release data was fitted into spherical matrix model also along-with first order, zero order and Higuchi equations.

\section{In- vitro cytotoxic activity}

The method used by Mehrotra and Pandit [10] was used here. The nanoparticle formulations used were of batches PD1 and PD4 with low particle size of different polymers and PD13 with low particle size containing Pluronic F68 surfactant.

\section{Stability studies at room temperature $\left(25^{\circ} \mathrm{C}\right)$ and at $45^{\circ} \mathrm{C}$}

The stability study was carried out of freshly prepared and dried under reduced pressure nanoparticles were conducted in sealed vials placed in stability chamber maintained at $25^{\circ} \mathrm{C}, 60 \% \mathrm{RH}$ and at $45^{\circ} \mathrm{C}$, $70 \% \mathrm{RH}$. The nanoparticles subjected to stability tests were analyzed over three month's period for drug content with a frequency of one month sampling. Three formulations were used for study namely formulation PD1 which was basic formulation, formulation PD4 with different polymer i.e. PDLG 7502 and formulation PD13 with different stabilizer i.e. $3 \% \mathrm{w} / \mathrm{v}$ Pluronic F68 producing smaller particle size.

\section{In-vivo biodistribution study in albino mice}

Healthy albino mice $(20 \pm 5 \mathrm{~g})$ were used for study. The animals were divided into groups of six mice each. Then $0.1 \mathrm{ml}$ of the suspension in buffer solution (PBS pH 7.4), containing lomustine, lomustine-loaded nanoparticles of different batches: PD1, PD4, PD13 (40 mg/kg lomustine equivalent) and buffer solution were intravenously administered into the mice through tail vein, separately in different groups. At predetermined time intervals $(12,24,36$ and $48 \mathrm{~h}$ ) blood samples ( $1 \mathrm{ml}$ in duplicate) were collected from the carotid artery, placed into heparinized test tubes and centrifuged to get corresponding plasma samples. Afterward, the animals were dissected and each tested organ (liver, lung, kidney, spleen heart) was excised. Blood was centrifuged in eppendorf tubes at $4^{\circ} \mathrm{C}$ (15000 rpm for $10 \mathrm{~min}$ ), and plasma was collected. Organs of interest (lung, kidney) were washed with $0.9 \%(\mathrm{w} / \mathrm{v})$ saline, blotted dry and were stored at $-80^{\circ} \mathrm{C}$ until analysis for lomustine. Aliquots of harvested organs $(80-150 \mathrm{mg})$ were homogenized in saline and acidified to $\mathrm{pH} 3.0$ with acetic acid. Both the plasma samples and tissue homogenates were stored at $4^{\circ} \mathrm{C}$ for $12 \mathrm{~h}$. Then tissue homogenates and plasma samples were treated with two volumes of cold mixture of acetonitrile/methanol $(1 / 1 \mathrm{v} / \mathrm{v})$ to precipitate proteins and extract lomustine. The obtained suspensions were centrifuged at $15,000 \mathrm{rpm}$ for $10 \mathrm{~min}$, and $20 \mu \mathrm{l}$ of the clear supernatant was injected into the HPLC system to determine the concentration of lomustine. Mobile phase was a mixture of acetonitrile/ water/methanol, 48:41:11v/v/v. For histological tissue analysis, kidney and lungs were fixed in neutral buffered $10 \%$ formalin, processed by standard methods in paraffin, sectioned at 5-10 $\mu \mathrm{m}$, and stained with hematoxylin and eosin (H\&E).

\section{Statistical analysis}

It's done a same analysis as published by Mehrotra and Pandit [10]. Mean values of nanoparticle size, polydispersity index, encapsulation efficiency and cytotoxic activity were compared.

\section{Results and Discussion}

\section{Saturation solubility of drug in stabilizer, surfactant, polymer and phosphate buffer}

Observed saturation solubility of lomustine in different solutions is shown in Figure 4. The experimental values are the average of three replicates and standard deviations did not exceed $3 \%$ of the mean value. The higher solubility of lomustine in phosphate buffer saline (PBS) $\mathrm{pH} 7.4$ could be attributed to its reported solubility in saline solution [11]. The saturation solubility of lomustine was significantly higher in PURASORB PDLG5002 solution (1\%w/v), PURASORB PDLG7502 solution $(1 \% \mathrm{w} / \mathrm{v})$ which was a result of its reported solubility in acetone.

\section{Differential Scanning Calorimetry (DSC)}

Thermogram of drug, polymer, and 1:1 physical mixture of drug: polymer was observed. There was no interaction between polymer and drug, a sharp endotherm of drug at $90.06^{\circ} \mathrm{C}$ and $91^{\circ} \mathrm{C}$ was observed with
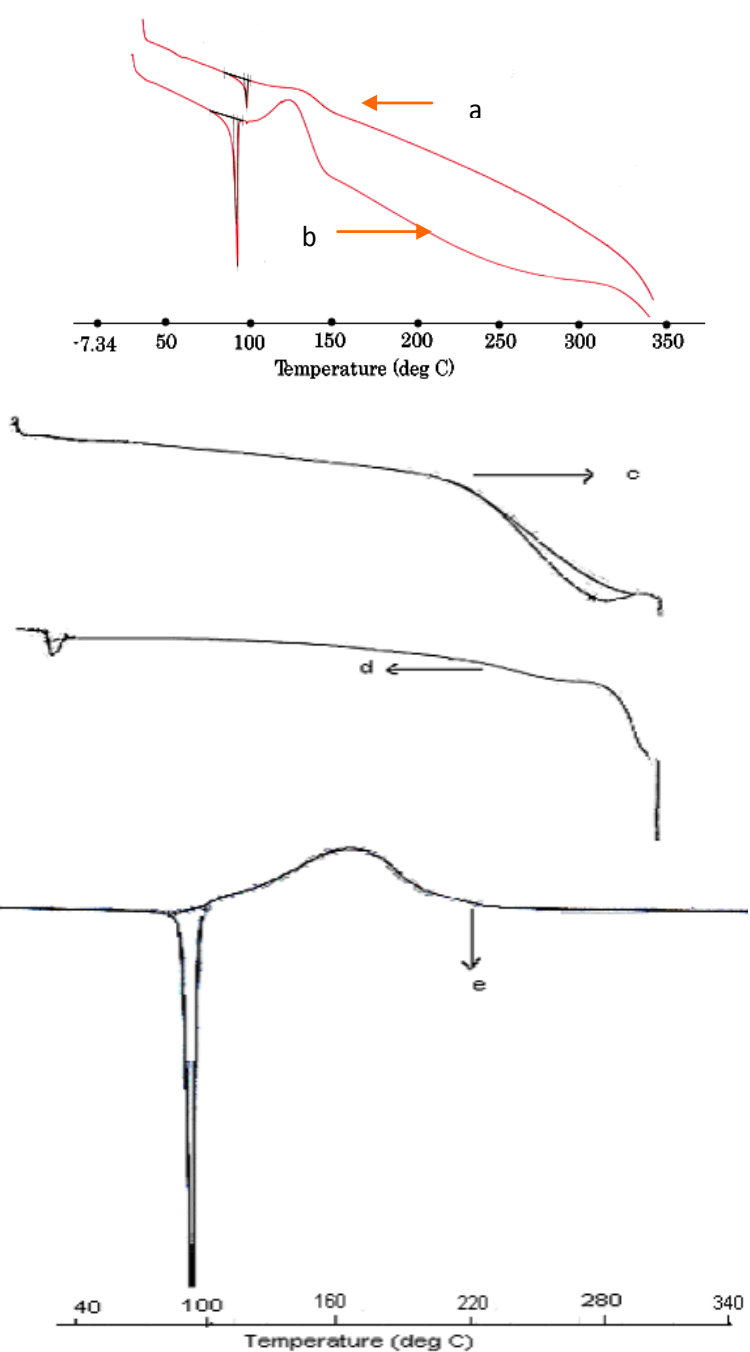

Figure 1: DSC thermogram of lomustine: PLGA physical mixture. a: endotherm of sample mixture 1:1 lomustine: PDLG 5002, b: endotherm of sample mixture 1:1 lomustine: PDLG 7502. c: polymer PDLG 5002, d: polymer 7502 , e: lomustine. 
Citation: Mehrotra A, Pandit JK (2015) Preparation and Characterization and Biodistribution Studies of Lomustine Loaded PLGA Nanoparticles by Interfacial Deposition Method. J Nanomed Nanotechnol 6: 328. doi:10.4172/2157-7439.1000328

mixture of polymer PDLG 5002 and polymer PDLG 7502 respectively. DSC curves are shown in Figure 1.

In this study, the physical interaction between lomustine and PLGA present in the 1:1 physical mixtures was studied. In Figure 1e, thermogram of pure lomustine showed an endotherm with sharp melting point. In Figure 1c and 1d pure PLGA polymer PDLG 5002 and PDLG 7502 exhibited relatively distorted thermal transition, confirming the amorphous nature of PLGA. From DSC tracings of the corresponding 1:1 physical mixtures of lomustine: PLGA (Figure 1a and $1 \mathrm{~b}$ ) a sharp endotherm can be easily attributed to the melting of crystalline lomustine that persisted in the physical mixture without any interaction.

\section{Infrared spectroscopic analysis}

The infrared spectroscopic analysis was performed to observe possible interactions between drug and polymer and to complement the results from DSC. The IR spectra for both drug and polymer and physical mixtures of drug: polymer shown in Figure 2a-d.

The characteristic bands observed from the IR data of lomustine included characteristic ring absorption of cyclohexyl ring of lomustine at $1533 \mathrm{~cm}^{-1}$ and $1490 \mathrm{~cm}^{-1}, \mathrm{C}=\mathrm{O}$ str. at $1703 \mathrm{~cm}^{-1}$ (Figure 2a). From the IR data of PLGA the characteristic absorption bands at $1733 \mathrm{~cm}^{-1}$ an ester group, $846 \mathrm{~cm}^{-1} \mathrm{C}$-C str. vibrations were identified (Figure 2b).

In the FTIR spectrum of 1:1 physical mixture of lomustine: PLGA (PDLG 5002 and 7502) the characteristic IR spectra were very similar, showing all the bands of the functional groups of lomustine and PLGA identified in the isolated compounds. The maintenance of these characteristic bands of both the drug and PLGA polymer, as well as the absence of new IR bands, indicates that there was no chemical interaction between the lomustine and the PLGA, demonstrating that lomustine does not react with the polymer and is only dissolved in the PLGA polymeric matrix. Any interaction present in physical mixture was tested in the thermal analysis experiments also. In fact, since both the drug and the polymer have similar lipophilicity character a homogeneous distribution of the molecular drug into the matrix structure of the polymeric nanoparticles could be expected.

\section{Particle size and zeta potential}

The nanoparticles were of nanometric size with homogeneous and a narrow particle size distribution and negative zeta potential (Table 2). Smaller particles were obtained when organic phase volume was increased to $20 \mathrm{ml}$ and $30 \mathrm{ml}$ and with $50 \mathrm{ml}$ of 3\%w/v pluronic F68 surfactant content.

This preparation method is applied to types of PLGA polymers, which varied in copolymer ratio lactic acid: glycolic acid (L/G). The difference in $L / G$ ratio did not have a significant influence on the average size, only slight increase was observed in size. This was in agreement with findings of Jiang et al. [12]. This could be regarded as a technical advantage of the interfacial deposition method, since the formation of nanoparticles would not be influenced by such factors as the $\mathrm{L} / \mathrm{G}$ ratio and the polymer species.

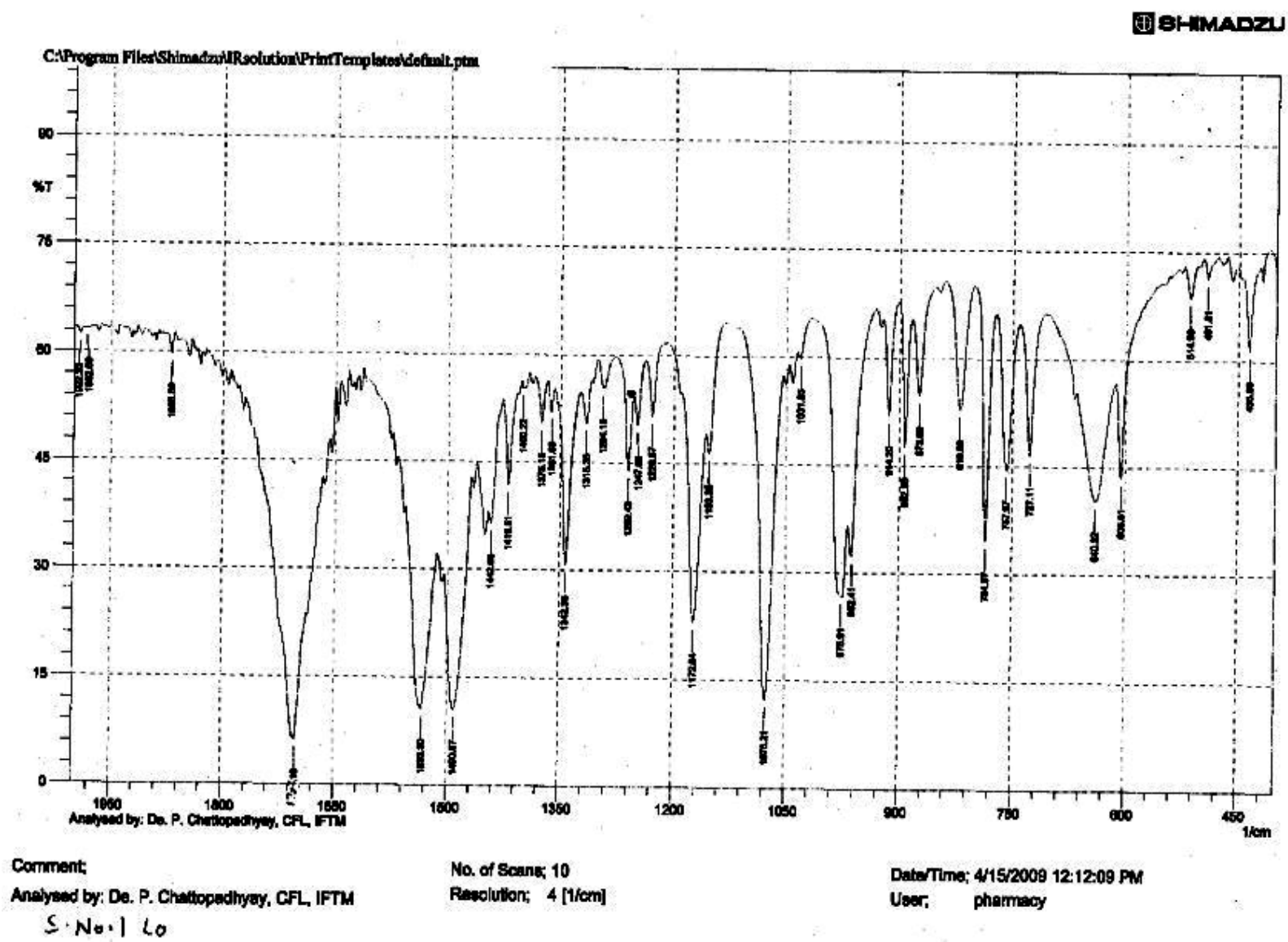

Figure 2a: FTIR spectrum of lomustine. 
Citation: Mehrotra A, Pandit JK (2015) Preparation and Characterization and Biodistribution Studies of Lomustine Loaded PLGA Nanoparticles by Interfacial Deposition Method. J Nanomed Nanotechnol 6: 328. doi:10.4172/2157-7439.1000328

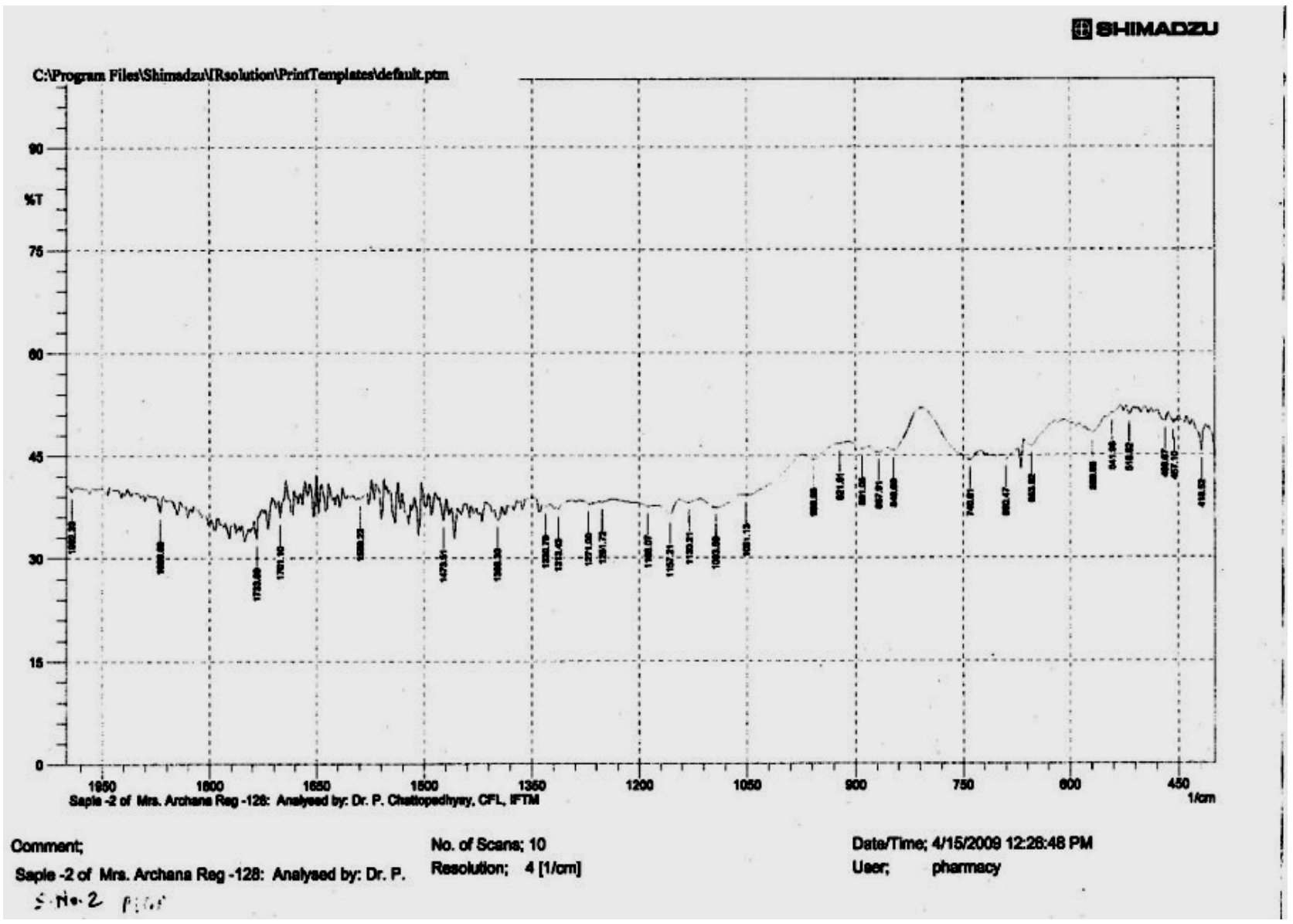

Figure 2b: FTIR spectrum of polymer PLGA 5002.

Pluronic F68 incorporated was oriented at organic solvent/water interface to reduce efficiently the interfacial tension, which resulted in significant increase in the net shear stress at a constant energy density during phase mixing [13] and resultant emulsification and promoted the formation of smaller emulsion droplets. Thus, the mean diameter of nanoparticles decreased with the presence and increase of Pluronic F68 concentration.

Acetone is a freely water-miscible organic solvent. When organic phase volume increased, the rapid dispersion of considerable amount of acetone into the external aqueous phase contributed to a significant decrease of the interfacial tension, thereby decreasing the particle size.

The negative charge on the PLGA nanoparticles was attributed to the presence of uncapped end ionized carboxyl groups of the polymer at the particle surface [14]. PVA has been extensively used as a promising stabilizer for PLGA nanoparticles. The mechanism of PVA binding with PLGA has been proposed to be due to the interpenetration of PVA and PLGA molecules during nanoparticle formation [15]. The presence of PVA formed a stable coating network on the polymer surface. This network shielded the surface charge and moved the shear plane outward from the particle surface, which resulted consequently in a slightly negative zeta potential. Despite this comparatively weak zeta potential, the nanoparticles stabilized by the layers of PVA surrounding the nanoparticles by steric hindrance.

The reduction seen in zeta potentials by using pluronic F68 surfactant was due to the fact that the coating layers shield the surface charge and move the plane of shear outwards from the particle surface $[16,17]$. Similarly the results obtained by Zou et al. [18] also correlate the zeta potential with shielding of the surface negative charge of PLGA.

\section{Scanning electron microscopy (SEM) of some critical batches}

The SEM analysis of nanoparticles of batches PD1, PD4, PD11, PD12, PD13, PD14, and PD15 was performed which shown smooth spherical particles as shown in Figure 3a-g respectively.

All the nanoparticles were spherical with smooth surface. No aggregation, separated particles with smooth surfaces confirmed suitability of different formulation parameters selected for preparation of nanoparticles. From the observed formulation parameters PVA was a critical factor affecting significantly a preparation process especially a purification and isolation step as there was observed some residual matter in SEM photomicrographs of formulations containing more amount of PVA than $50 \mathrm{ml}$ 1\%w/v PVA (Figure 3c and 3f). This might be attributed to increased viscosity of aqueous solution in presence of increased amount of PVA, which require extra washings of residue after centrifugation. Surfactant pluronic F68 in all concentrations used produced nanoparticles with smooth surfaces with method of interfacial deposition used.

\section{Encapsulation efficiency}

The effect of variable process parameters on entrapment efficiency is 
Citation: Mehrotra A, Pandit JK (2015) Preparation and Characterization and Biodistribution Studies of Lomustine Loaded PLGA Nanoparticles by Interfacial Deposition Method. J Nanomed Nanotechnol 6: 328. doi:10.4172/2157-7439.1000328

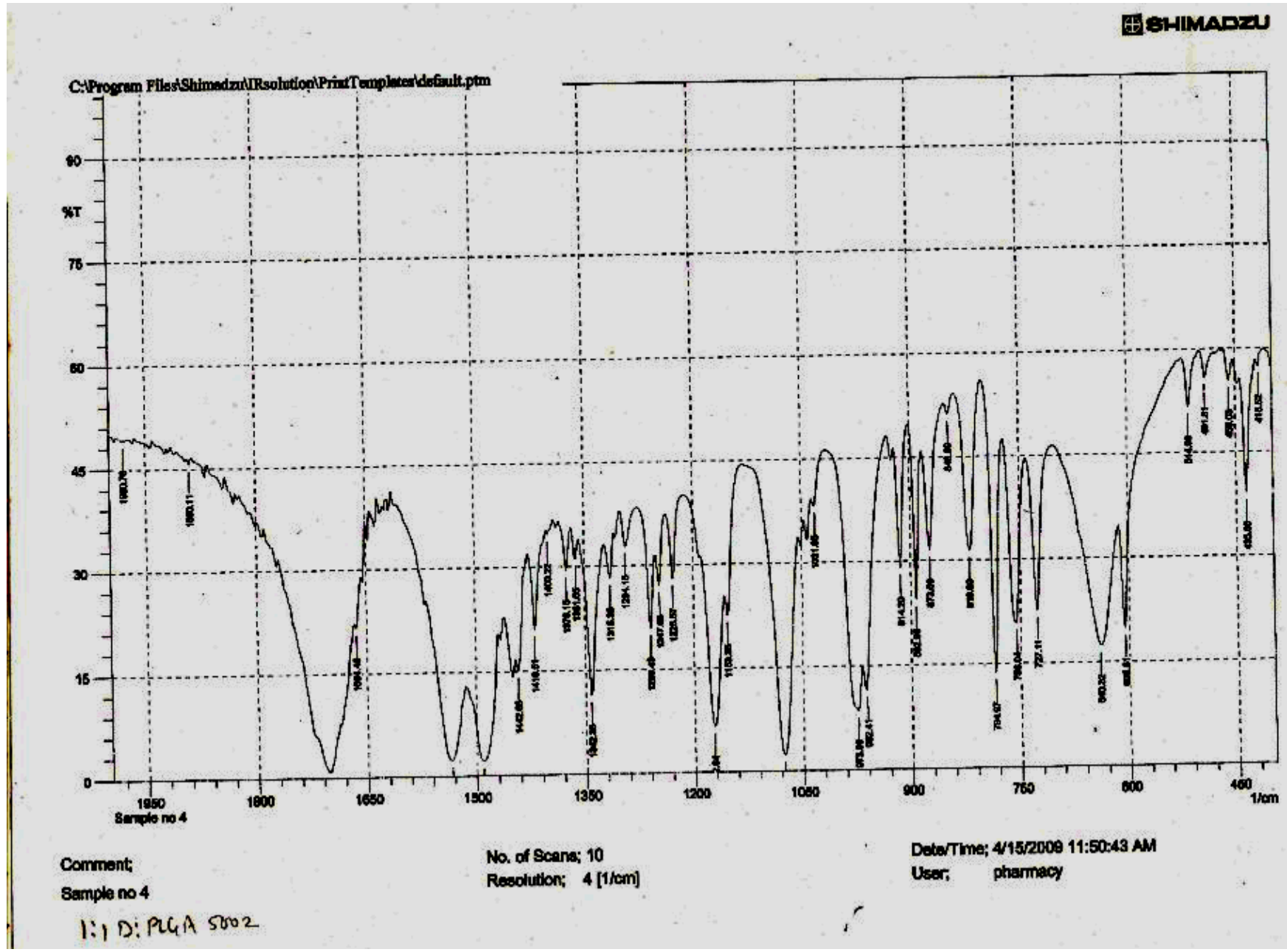

Figure 2c: FTIR spectrum of 1:1 physical mixture of lomustine: PLGA5002.

given in Table 2. Entrapment efficiency was increased significantly with increasing polymer concentration. It was increased slightly with using copolymer PDLG 7502 and increased significantly with increasing amount of lomustine $(\mathrm{p}<0.05)$. It was decreased with increasing amount of surfactant pluronic F68 from $1 \%$ to $3 \% \mathrm{w} / \mathrm{v}$.

Lomustine demonstrated the highest encapsulation efficiency in this process used. More lipophilic drugs do not suffer from the problems of leakage of drug to the external medium, resulting in improved drug content in the nanoparticles. The entrapment efficiency of drug in nanoparticles correlated well with its solubility in different stabilizer solutions [19]. Due to greater solubility of lomustine in pluronic F68 solution than in PVA solution, on mixing of two phase's lomustine migrates rapidly to the external phase resulting in low entrapment, while in PVA solution, the hydrophobic interaction of the lomustine and polymer supersedes due to the lower solubility of lomustine in PVA solution, resulting in higher entrapment efficiency. In these lomustine nanoparticles, hydrophobicity of drug and relative proportions of drug/ polymer/solvent might be one of the causes of high encapsulation efficiency [20].

Danhier et al. [21] observed that nanoparticles prepared with interfacial deposition/nanoprecipitation method could achieve higher encapsulation efficiency than with the simple emulsion technique.
Thus the process itself is beneficial for encapsulation efficiency of hydrophobic drugs. Addition of droplet stabilizer less polar for drug further showed synergistic effect.

As the amount of lactide of PLGA increased, encapsulation efficiency of lomustine increased slightly. With the increase of L: G ratio of PLGA, the interaction or affinity of drug probably increased [13-22].

The encapsulation efficiency of lomustine increased significantly $(p<0.05)$ with the increase of PLGA concentration. This phenomenon probably resulted from the increase of viscosity. Increasing viscosity could increase the drugs resistance diffusional into the aqueous phase and thus enhance the drugs incorporation into nanoparticles. Additionally, larger nanoparticles had higher drug entrapment efficiencies.

The slightly increased encapsulation efficiency with increase of PVA concentration was probably caused by the increase in particle size. Moreover, with the increase of pluronic F68 concentration, more molecules of lomustine might be partitioned out rapidly into the aqueous phase during phase mixing procedure and less drug molecules remained in emulsion droplets to interact with PLGA molecules, hence decreasing the encapsulation efficiencies.

The increase of the lomustine content resulted in a significant increase 


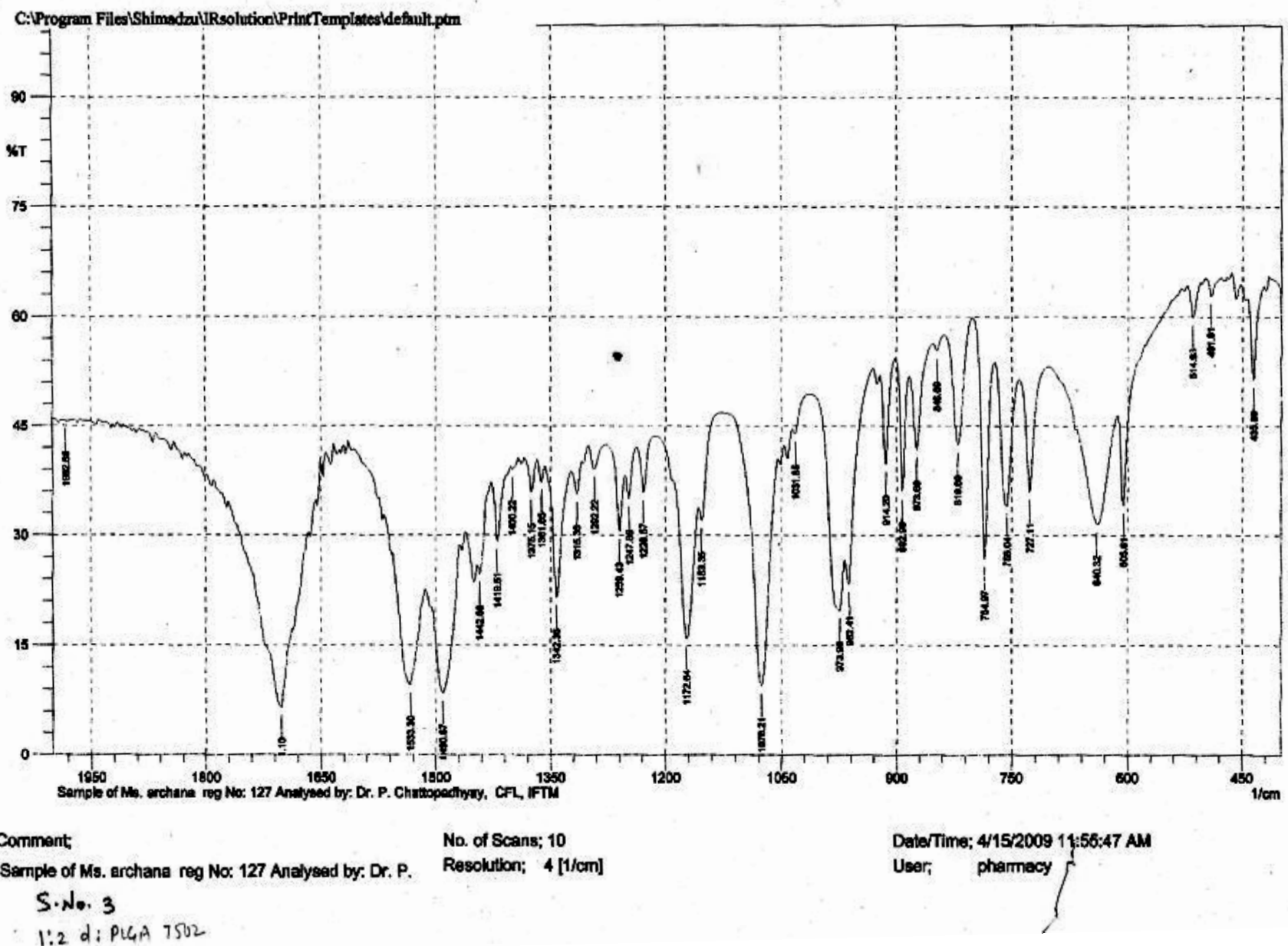

Figure 2d: FTIR spectrum of 1:1 physical mixture of lomustine:PLGA 7502.

in encapsulation efficiency $(\mathrm{p}<0.05)$ as the lomustine concentration in the organic phase increased and then more drug molecules could interact with PLGA molecules, resulting in the increased encapsulation of lomustine. As the initial lomustine amount increased, the amount of lomustine partitioned into the aqueous phase probably reduced during phase mixing procedure. Thus more lomustine molecules could remain in emulsion droplets to interact with PLGA molecules and the encapsulation of lomustine could be further enhanced.

When aqueous phase volume increased, the amount of lomustine precipitated in the aqueous phase resulted in less lomustine retention in the internal phase to interact with PLGA molecules and then shown lower encapsulation efficiency.

The encapsulation efficiency of lomustine increased first with the increase of organic phase volume $(p<0.05)$ then it decreased. This occurred possibly because the change of acetone volume affected the partition of drug in the organic phase. The hydrophobic molecule lomustine was easy to dissolve in acetone. When acetone volume increased, more lomustine molecules were carried into the aqueous phase or at organic solvent/water interface by considerable amount of acetone; and thus less lomustine molecules remained in the internal phase to interact with PLGA molecules and lower entrapment efficiency was obtained. Afterwards, further increase in acetone volume increased the partition of lomustine in the organic phase. Hence, more lomustine molecules interacted with PLGA molecules in the internal phase and enhanced the encapsulation.

\section{In- vitro drug release}

The percentage drug release of lomustine in Franz diffusion cell was complete. The in vitro release behaviour of lomustine from PLGA nanoparticles; from the polymer matrix exhibited a fast initial release during the first $24 \mathrm{~h}$ followed by a slower and continuous sustained release over $48 \mathrm{hr}$.

The release rate of the lomustine from the nanoparticles and its appearance in the dissolution medium was governed by the partition coefficient of the drug between the polymeric phase and the aqueous environment in the dialysis bag and by the diffusion of the drug across the membrane as well. The dialysis bag retained the nanoparticles and allowed the diffusion of the drug immediately into the receiver compartment [23]. The biphasic release profile, with an initial burst of drug release attributed to surface associated drug, followed by a phase of slower release as drug entrapped in the nanoparticles diffuses out into the release medium.

Also, the effect of different formulation variables on drug release was observed. Effect of polymer amount on drug release profile of 
Citation: Mehrotra A, Pandit JK (2015) Preparation and Characterization and Biodistribution Studies of Lomustine Loaded PLGA Nanoparticles by Interfacial Deposition Method. J Nanomed Nanotechnol 6: 328. doi:10.4172/2157-7439.1000328

Page 8 of 13
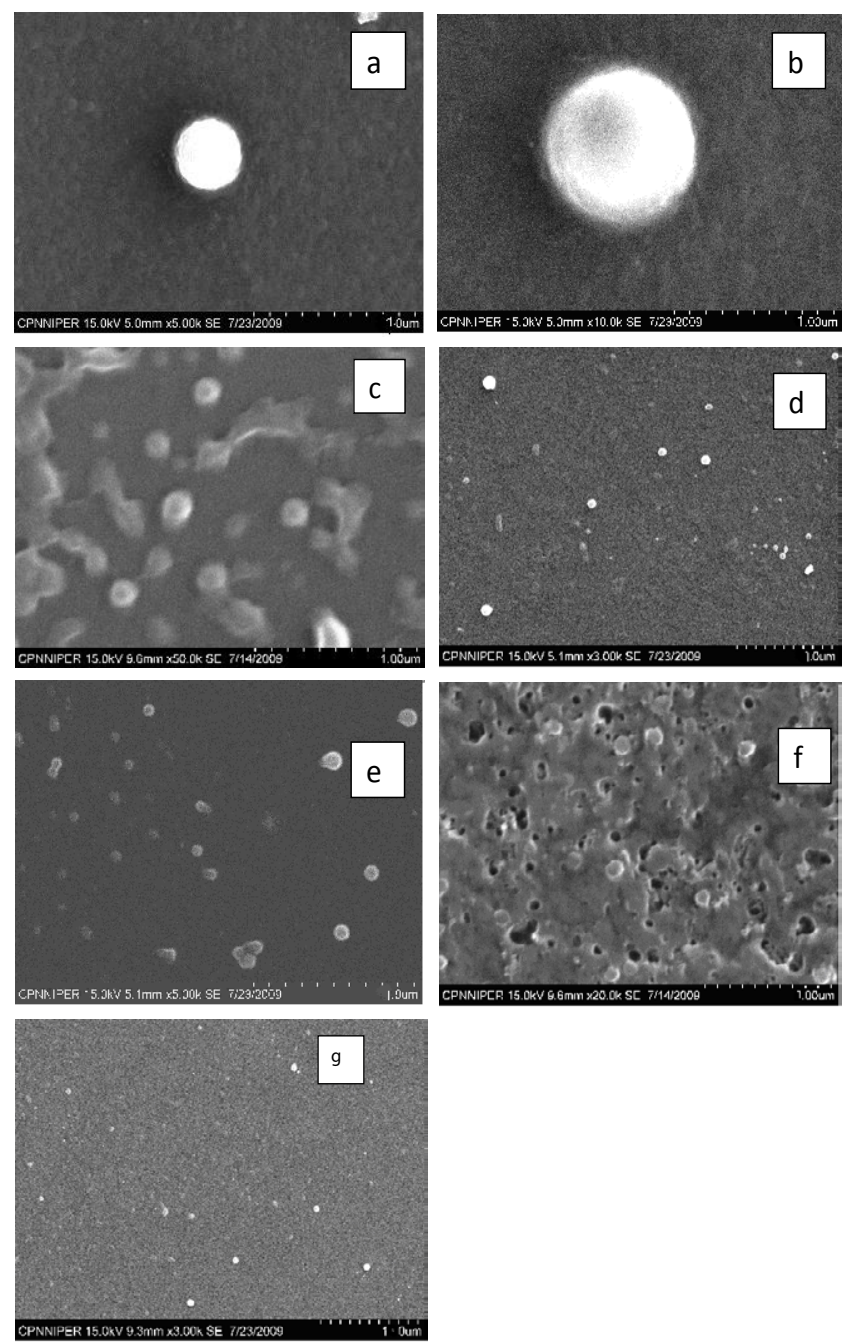

Figure 3: SEM Images of lomustine loaded nanoparticles. (a) Nanoparticles prepared using $50 \mathrm{ml}$ of $1 \% \mathrm{w} / \mathrm{v}$ PVA solution and polymer PDLG 5002 (Basic, formmualtion PD1). Magnification 5.00k SE. Magnification 10.0 k SE. (b) Nanoparticles prepared using $50 \mathrm{ml}$ of $1 \% \mathrm{w} / \mathrm{v}$ PVA solution and polymer PDLG 7502 (Batch PD4). (c) Nanoparticles prepared using $50 \mathrm{ml}$ of (Batch PD11).Magnification $5.00 \mathrm{k}$ SE3\%w/v PVA. (d) Nanoparticles prepared using $50 \mathrm{ml}$ of $1 \% \mathrm{w} / \mathrm{v}$ pluronic F68 (Batch PD12). Magnification $3.00 \mathrm{k}$ SE. (e) Nanoparticles prepared using $50 \mathrm{ml}$ of $3 \% \mathrm{w} / \mathrm{v}$ pluronic F68 (Batch PD13). Magnification 5.00 k SE. (f) Nanoparticles prepared using $100 \mathrm{ml}$ of $1 \%$ w/v PVA solution (Batch PD14). Magnification 20.0 k SE. (g) SEM image of nanoparticles prepared using $100 \mathrm{ml}$ of 1\% pluronic F68 (Batch PD15) Magnification $3.00 \mathrm{k} \mathrm{SE}$

lomustine (Figures 5 and 6) with respect to basic formulation showed that the drug release was slow as the amount of polymer was increased. This was because of increased viscosity and diffusion path. Molecular weight and crystallinity of the polymer influence drug release and degradation of the nanoparticles [2]. Also this drug release mirrors polymer mass loss and was therefore dependent on polymer composition and molecular weight, the higher the molecular weight and lactide content of the polymer the longer the lag time (reflected in a large $t_{\max }$ ) before the commencement of the polymer degradation controlled phase [24].

When organic phase volume was increased drug release rate was increased (Figure 7). This was due to increased solubility of lomustine in increased volume of organic solvent resulting increased diffusivity and hydrodynamics at the interface.

When drug loading was increased drug released rate was increased (Figure 8). The reason proposed by Corrigan and Li [24] for higher drug loading giving more rapid release, was that a high density of interconnecting channels ('active sites') increase polymer permeability in the presence of drug and that result in increased PLGA degradation and erosion. In addition the polymer degradation rate is influenced by the presence of drug which may interact [25] and cause plasticization of the polymer, a lowering of the $T \mathrm{~g}$ and more rapid polymer degradation and hence drug release.

A slight increase in drug release was observed when concentration of surfactant Pluronic F68 was increased (Figure 9).

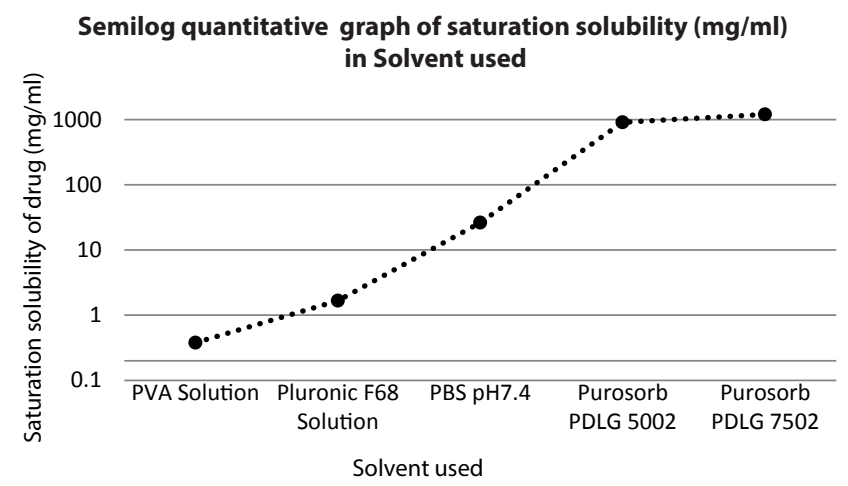

Figure 4: Saturation solubility of lomustine in different solvent solutions.

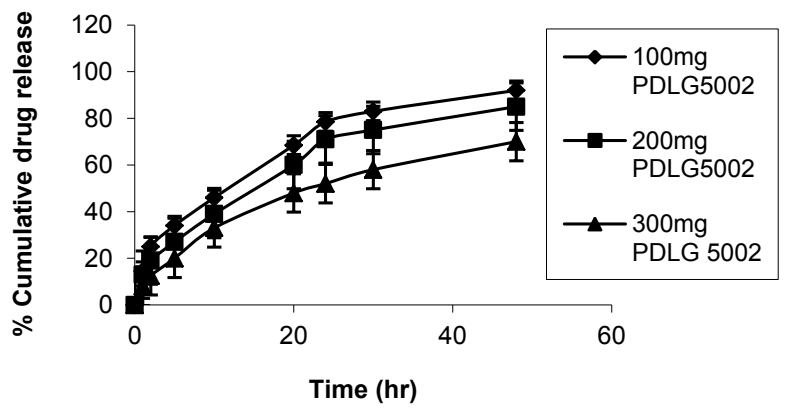

Figure 5: Drug release profile of lomustine from nanoparticles as a function of polymer amount.

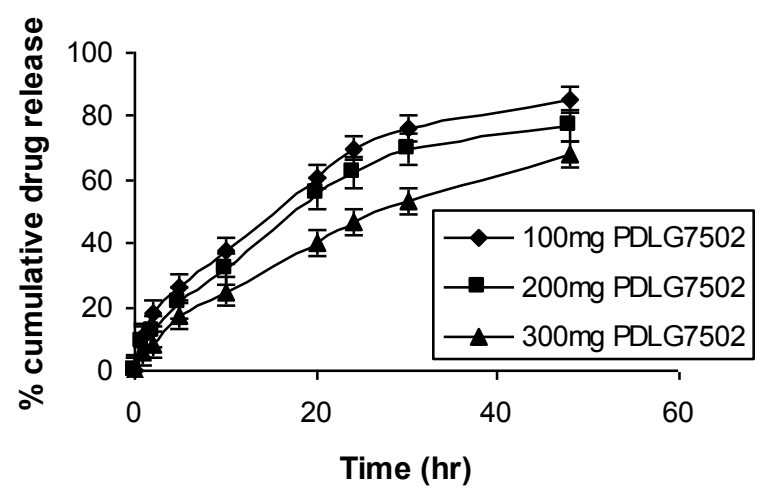

Figure 6: Drug release profile of lomustine from nanoparticles as a function of polymer amount. 
Citation: Mehrotra A, Pandit JK (2015) Preparation and Characterization and Biodistribution Studies of Lomustine Loaded PLGA Nanoparticles by Interfacial Deposition Method. J Nanomed Nanotechnol 6: 328. doi:10.4172/2157-7439.1000328

Page 9 of 13

\begin{tabular}{|c|c|c|c|c|c|c|}
\hline S.No & Batch code & Particle size $(\mathrm{nm})$ (Mean $\left.\pm \mathrm{SD}^{\star}\right)$ & $\begin{array}{l}\text { Polydispersity index } \\
\text { (PDI) (Mean } \pm \text { SD*) }\end{array}$ & $\begin{array}{l}\text { Zeta potential }(\mathrm{mV}) \\
\left(\text { Mean } \pm \mathrm{SD}^{*}\right)\end{array}$ & Drug content (mg) & $\%$ EE (Mean \pm SD*$\left.^{*}\right)$ \\
\hline 1 & PD1 & $296.9 \pm 12.2$ & $0.135 \pm 0.013$ & $-0.964 \pm 0.029$ & 0.87 & $87 \% \pm 0.76$ \\
\hline 2 & PD2 & $316.2 \pm 13.4$ & $0.128 \pm 0.009$ & $-2.09 \pm 0.063$ & 0.92 & $92.4 \% \pm 0.70$ \\
\hline 3 & PD3 & $335.8 \pm 10.6$ & $0.122 \pm 0.016$ & $-8.14 \pm 0.076$ & 0.98 & $98.1 \% \pm 0.67$ \\
\hline 4 & PD4 & $318.9 \pm 14.3$ & $0.136 \pm 0.012$ & $-2.06 \pm 0.012$ & 0.88 & $88.3 \% \pm 0.81$ \\
\hline 5 & PD5 & $344.5 \pm 13.6$ & $0.127 \pm 0.011$ & $-1.29 \pm 0.034$ & 0.93 & $93 \% \pm 0.66$ \\
\hline 6 & PD6 & $370.3 \pm 11.4$ & $0.138 \pm 0.006$ & $-0.61 \pm 0.012$ & 0.98 & $98.6 \% \pm 1.02$ \\
\hline 7 & PD7 & $227.3 \pm 13.5$ & $0.131 \pm 0.016$ & $-0.471 \pm 0.008$ & 0.85 & $85 \% \pm 0.86$ \\
\hline 8 & PD8 & $198.5 \pm 11.4$ & $0.110 \pm 0.014$ & $-0.238 \pm 0.017$ & 0.80 & $80.3 \% \pm 0.84$ \\
\hline 9 & PD9 & $304.2 \pm 12.5$ & $0.117 \pm 0.011$ & $-7.78 \pm 0.121$ & 4.8 & $96 \% \pm 0.82$ \\
\hline 10 & PD10 & $307.8 \pm 11.2$ & $0.124 \pm 0.008$ & $-8.2 \pm 0.018$ & 9.95 & $99.5 \% \pm 0.75$ \\
\hline 11 & PD11 & $334.6 \pm 10.7$ & $0.156 \pm 0.007$ & $-4.44 \pm 0.098$ & 0.88 & $88 \% \pm 0.63$ \\
\hline 12 & PD12 & $177.2 \pm 14.1$ & $0.135 \pm 0.018$ & $-4.3 \pm 0.26$ & 0.81 & $81 \% \pm 0.85$ \\
\hline 13 & PD13 & $167.9 \pm 11.5$ & $0.143 \pm 0.011$ & $-5.1 \pm 0.312$ & 0.79 & $79 \% \pm 0.68$ \\
\hline 14 & PD14 & $298.4 \pm 10.1$ & $0.192 \pm 0.007$ & $-6.63 \pm 0.148$ & 0.86 & $88 \% \pm 0.65$ \\
\hline 15 & PD15 & $197.9 \pm 13.2$ & $0.278 \pm 0.007$ & $-5.8 \pm 0.207$ & 0.85 & $85 \% \pm 0.9$ \\
\hline
\end{tabular}

$\mathrm{n}=3, \mathrm{SD}^{*}=$ Standard deviation

Table 2: Effect of various process parameters on particle size, size distribution, zeta potential, drug content and encapsulation efficiency.

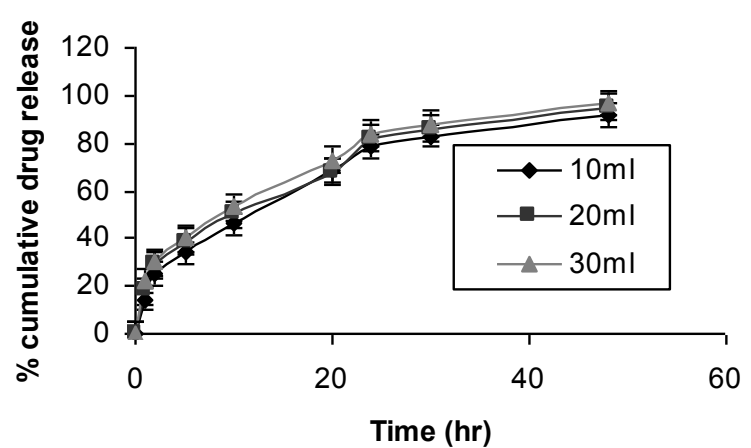

Figure 7: Drug release profile of lomustine from nanoparticles as a function of organic phase volume.

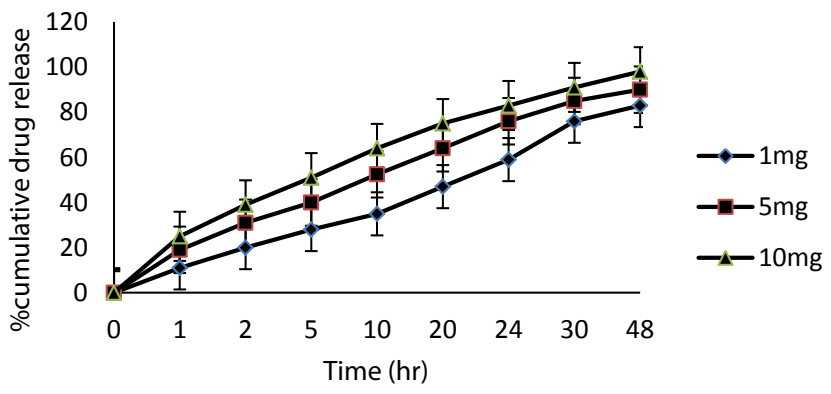

Figure 8: Drug release profile of lomustine from nanoparticles as a function of lomustine content.

This was attributed to the high concentration of drugs dispersed, which effectively increases the proportion of drug linked to the particle surface-liquid interface.

The nanoparticle size was also associated with changes in drug release kinetics. The smaller sized nanoparticles prepared with lower amounts of PLGA showed higher drug release rates. This release behaviour may be explained by a corresponding increase in the total nanoparticles surface, resulting in a larger drug fraction exposed to the leaching medium. Smaller nanoparticles size lead to shorter average diffusion path of the matrix-entrapped drug molecules [26]. The diffusion distances encountered in the particles were small which allowed drug trapped in the core to rapidly diffuse out and also for the release medium to diffuse in.

\section{Mechanism of drug release}

The correlation coefficients $\left(\mathrm{r}^{2}\right)$ values for a plot of Higuchi's equation were more linear than for other plots. The values were in the range of 0.9758 to 0.9976 , which was always higher than other equations indicating square-root time dependant release kinetics of lomustine from the lomustine loaded nanoparticles. The release exponents (n) were $<1.0$ indicating non-fickian release mechanism.

\section{Stability study}

Nanoparticles were studied for stability at room temperature i.e. $25^{\circ} \mathrm{C} / 60 \% \mathrm{RH}$ and at $45^{\circ} \mathrm{C} / 70 \% \mathrm{RH}$ for three months and drug content was found as shown in Table 3.

After storage for 3 months at $25^{\circ} \mathrm{C} / 60 \% \mathrm{RH}$, nanoparticles displayed no significant change $(\mathrm{p}>0.05)$ of drug content for all the three tested nanoparticle batches. At $45^{\circ} \mathrm{C} / 70 \% \mathrm{RH}$ the drug content was maintained and not significantly $(\mathrm{p}>0.05)$ affected for one month, after 3 months nanoparticles drug content was slightly affected during storage. This result showed that PLGA nanoparticles of lomustine prepared possessed good storage stability at $25^{\circ} \mathrm{C} / 60 \% \mathrm{RH}$, due to an adequate proportion of different constituents in the preparations. At $45^{\circ} \mathrm{C} / 70 \%$ RH nanoparticles with higher concentration of pluronic F68 preserved the content of a thermolabile drug lomustine, proving it a suitable stabilizer.

\section{In-vitro anticancer activity}

The percent viable cells were reduced with nanoparticles formulation as shown in Figure 10. In-vitro cytotoxic activity tested showed that cell viability was decreased significantly $(p<0.05)$ by lomustine nanoparticles and percent cell viability and $\mathrm{IC}_{50}$ values at 24 $\mathrm{h}$ incubation are given in Table 4.

Lomustine nanoparticles showed significant anticancer activity ( $p$ 
Citation: Mehrotra A, Pandit JK (2015) Preparation and Characterization and Biodistribution Studies of Lomustine Loaded PLGA Nanoparticles by Interfacial Deposition Method. J Nanomed Nanotechnol 6: 328. doi:10.4172/2157-7439.1000328

Page 10 of 13

\begin{tabular}{|c|c|c|c|c|c|c|c|c|}
\hline \multirow{3}{*}{ S.NO } & \multirow{3}{*}{ Batch code } & \multirow{3}{*}{$\begin{array}{c}\text { Initial Drug } \\
\text { Content }(\% w / w) \\
\left(\text { mean } \pm S D^{*}\right)\end{array}$} & \multicolumn{6}{|c|}{ Drug content $(\% w / w)$} \\
\hline & & & \multicolumn{2}{|c|}{ After one month } & \multicolumn{2}{|c|}{ After two months } & \multicolumn{2}{|c|}{ After three months } \\
\hline & & & $\begin{array}{c}\text { At } 25^{\circ} \mathrm{C} / 60 \% \text { RH } \\
\text { (mean } \pm \text { S.D*) }\end{array}$ & $\begin{array}{c}\text { At } 45^{\circ} \mathrm{C} / 70 \% \mathrm{RH} \\
\left(\text { mean } \pm \mathrm{SD}^{\star}\right)\end{array}$ & $\begin{array}{l}\text { At } 25^{\circ} \mathrm{C} / 60 \% \mathrm{RH} \\
(\text { mean } \pm \text { S.D } \\
* \text { ) }\end{array}$ & $\begin{array}{c}\text { At } 45^{\circ} \mathrm{C} / 70 \% \mathrm{RH} \\
\left(\text { mean } \pm \mathrm{SD}^{*}\right)\end{array}$ & $\begin{array}{c}\text { At } 25^{\circ} \mathrm{C} / 60 \% \text { RH } \\
\text { (mean } \pm \text { S.D*) }\end{array}$ & $\begin{array}{c}\text { At } 45^{\circ} \mathrm{C} / 70 \% \mathrm{RH} \\
\left(\text { mean } \pm \mathrm{SD}^{*}\right)\end{array}$ \\
\hline 1 & PD1 & $90 \pm 0.03$ & $90 \pm 0.01$ & $89 \pm 0.03$ & $89.9 \pm 0.05$ & $88.6 \pm 0.01$ & $89.9 \pm 0.02$ & $87.9 \pm 0.06$ \\
\hline 2 & PD4 & $90.6 \pm 0.05$ & $90.6 \pm 0.04$ & $90.3 \pm 0.04$ & $90.6 \pm 0.02$ & $90.0 \pm 0.04$ & $90.5 \pm 0.03$ & $89.8 \pm 0.02$ \\
\hline 3 & PD13 & $97 \pm 0.05$ & $97 \pm 0.02$ & $96.8 \pm 0.03$ & $97 \pm 0.04$ & $96.7 \pm 0.05$ & $97 \pm 0.03$ & $96.5 \pm 0.04$ \\
\hline
\end{tabular}

"n=3 SD: Standard deviation

Table 3: Drug content of nanoparticles stored at different temperatures for 3 months.

\begin{tabular}{|c|c|c|c|}
\hline S. No. & Formulation & Cytotoxicity $\left(\mathrm{IC}_{50} \mu \mathrm{g} / \mathrm{ml}\right)$ & $\%$ Cell viability \\
\hline 1 & Lomustine & $32 \pm 1.6$ & $28 \pm 0.98$ \\
\hline 2 & PD1 & $17 \pm 0.92$ & $21 \pm 1.3$ \\
\hline 3 & PD4 & $19 \pm 1.1$ & $26 \pm 0.86$ \\
\hline 4 & PD13 & $13 \pm 1.2$ & $18 \pm 0.71$ \\
\hline
\end{tabular}

Table 4: Cytotoxic activity of lomustine and nanoparticles of batch PD1, PD4 and PD13 against L132 cell line at $24 \mathrm{~h}$.

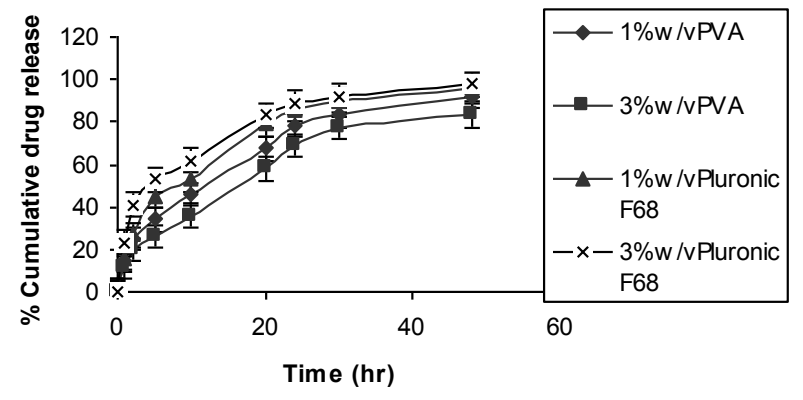

Figure 9: Drug release profile of lomustine from nanoparticles as a function of PVA and surfactant Pluronic F68 concentration.

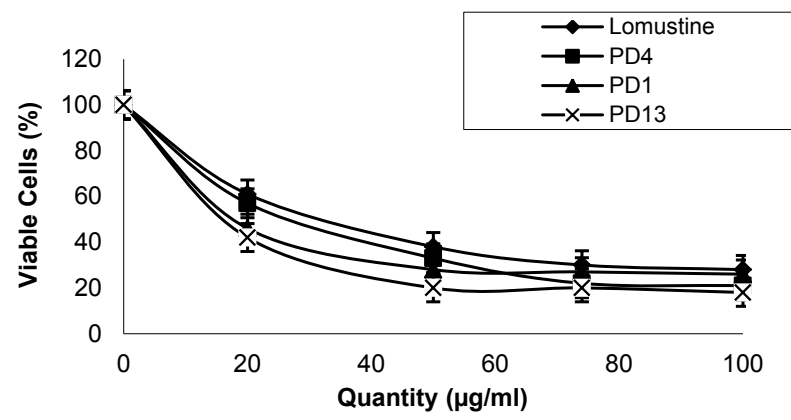

Figure 10: Viability of L132 cells with lomustine (A), nanoparticles of batch PD1 (B), nanoparticles of batch PD4 (C) and nanoparticles of batch PD13 (D).

$0.05)$ at concentration of $100 \mu \mathrm{g} / \mathrm{ml}$. The observed cytotoxic effect on cells probably arose from adhesion of solid nanoparticles to the cell surface and cell uptake of nanoparticles $[1,27,28]$. Surface properties of nanoparticles determine the performance of the nanoparticle system in the body, e.g. interactions with cell membranes followed by drug released inside the cells after cell uptake [2]. Thus the cytotoxicity of L132 cells mainly related to the released lomustine from lomustineloaded PLGA nanoparticles inside the cells after cell uptake. These suggested that the pharmacological activity of lomustine loaded PLGA nanoparticles was maintained and efficiently delivered lomustine to cells.

Biodistribution profile of lomustine and lomustine

\section{nanoparticles in mice}

There was fast decay of lomustine in plasma when lomustine solution was administered to mice (Figure 11) and at $24 \mathrm{~h}$ post administration, the lomustine concentration in plasma could not be detected.

The plasma concentration of lomustine after administration of nanoparticles was higher (Figure 11) and still showed lomustine concentration in plasma at $48 \mathrm{~h}$. The lomustine concentration profiles in plasma shown here indicate that lomustine level in blood could be maintained for an extended time period for nanoparticle sample due to the long circulating property of the polymeric nanoparticles. The higher plasma concentration of PD13 nanoparticles was due to the presence of Pluronic F68 layer on the surface, which shifted the shear plane of the diffusive layer to a larger distance. Surface modification or coating by biocompatible (hydrophilic) polymers improve uptake of nanoparticles and enhance stability. PEG, poloxamers and poloxamines are examples [2].

The distribution profiles of lomustine in lungs and kidney showed that compared with lomustine solution (Figure 12a), the biodistribution of lomustine was changed in the group treated with lomustine-loaded nanoparticles (batch PD1, PD4 and PD13 (Figure 12b-d respectively). Lomustine concentration in lung was elevated with the nanoparticles than with lomustine solution and it was in decreasing order with nanoparticles of batch PD13 > PD4 > PD1. Lomustine elimination was slow when nanoparticles were administered to mice as it was detected in kidney even after $48 \mathrm{~h}$ post administration as compared to lomustine solution.

Here, For nanoparticles taken up by opsonisation of the mononuclear phagocytic system (MPS) present in the liver, spleen, bone-marrow and kidney, instead of particle size, deformability of nanoparticles might be considered to understand their distribution in tissues and opsonization by MPS, because the real particle size can be changed against the deformability of particles (i.e. the mechanical strength, swelling behavior, capacity to undergo hydrolysis and subsequent biodegradation) in the blood stream.

Only a pulmonary toxicity of lomustine has been reported in literature at cumulative doses usually greater than $1.100 \mathrm{mg} / \mathrm{m}^{2}$. There is one report of pulmonary toxicity at a cumulative dose of only 600 $\mathrm{mg}$ [29]. Also as these nanoparticles are specifically fabricated for lung cancer application and to simulate in-vitro anticancer activity, the quantity of lomustine in lungs histopathologically studied alongwith clearance time and concentration. As these nanoparticles are meant for parenteral intravenous administration, it avoids absorption phase and first pass metabolism effect. So it surpasses liver studies histopathologically.

Histopathological examination of the lung and kidney of albino mice was carried out to study the presence of any toxicity after administration of lomustine nanoparticles as compared to vehicle and lomustine solution at $24 \mathrm{~h}$ post administration. 
Citation: Mehrotra A, Pandit JK (2015) Preparation and Characterization and Biodistribution Studies of Lomustine Loaded PLGA Nanoparticles by Interfacial Deposition Method. J Nanomed Nanotechnol 6: 328. doi:10.4172/2157-7439.1000328

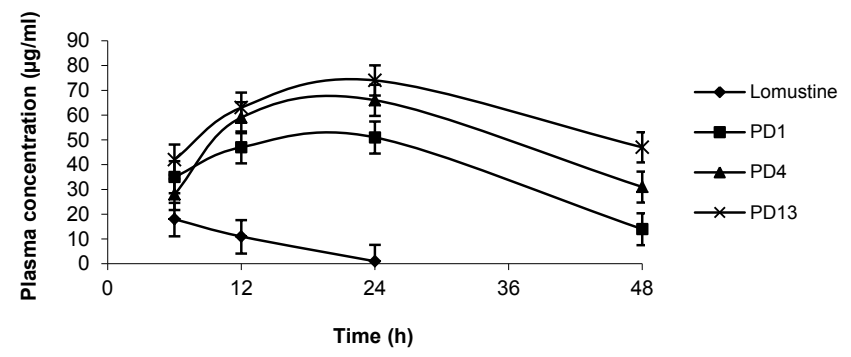

Figure 11: Lomustine concentration in plasma in albino mice at various time intervals after administration of lomustine solution and nanoparticles of batch PD1, PD4, PD13.

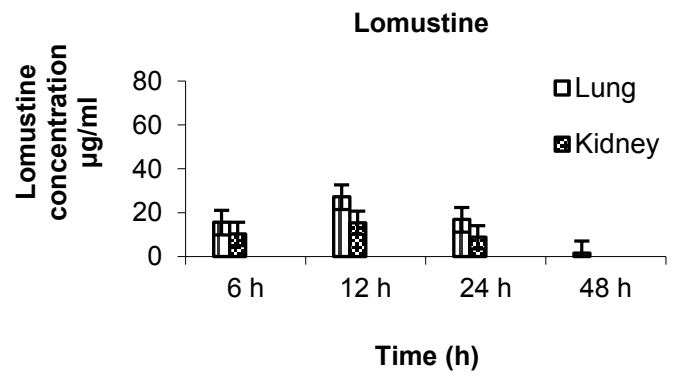

Figure 12a: Biodistribution of lomustine in albino mice after administration of lomustine solution.

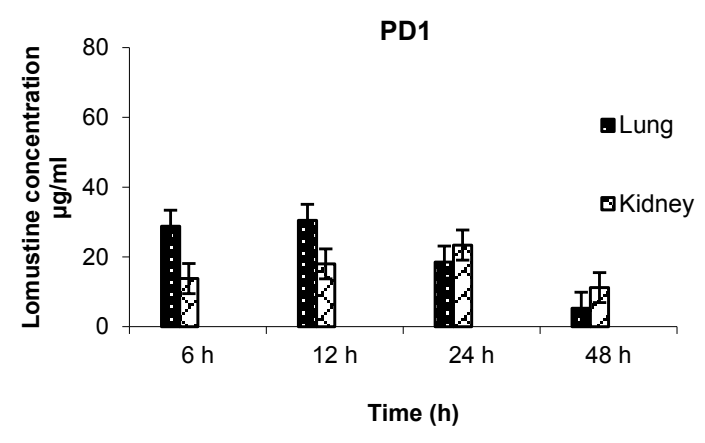

Figure 12b: Biodistribution of lomustine in albino mice after administration of lomustine nanoparticles batch PD1.

\section{PD4}

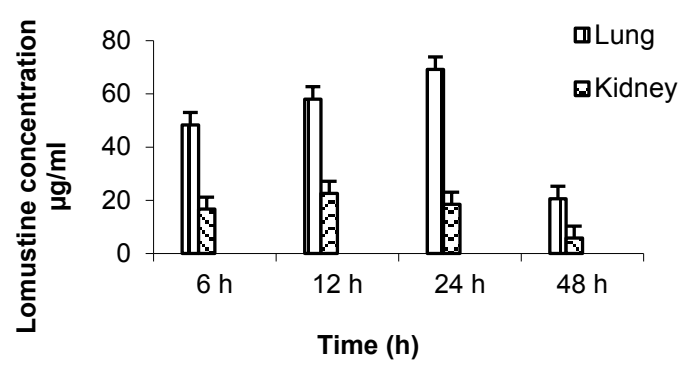

Figure 12c: Biodistribution of lomustine in albino mice after administration of lomustine nanoparticles batch PD4.

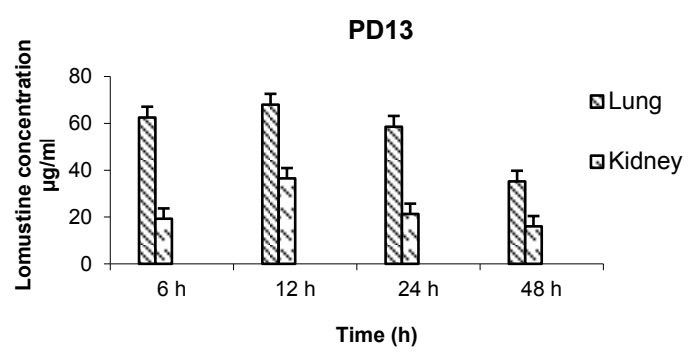

Figure 12d: Biodistribution of lomustine in albino mice after administration of lomustine nanoparticles batch PD13.

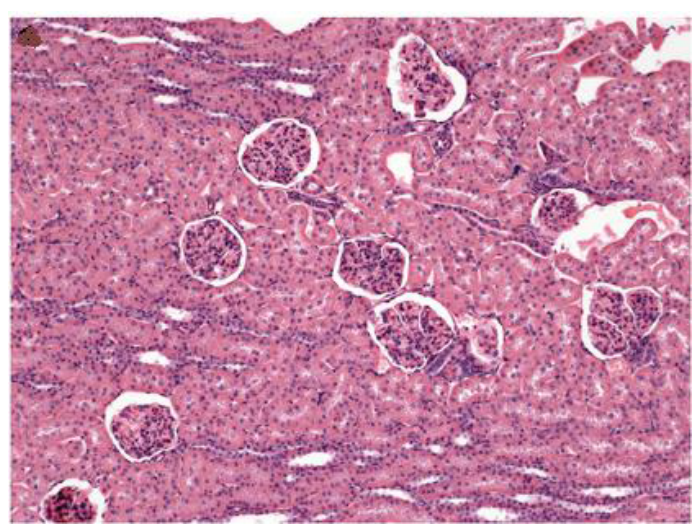

Figure 13a: Photomicrograph of H\&E stained histological section (magnification $10 \times 10$ ) showing the effect of vehicle PBS $\mathrm{pH} 7.4$ on the albino mice kidney $24 \mathrm{~h}$ after intravenous injection $0.1 \mathrm{ml} / \mathrm{kg}$.

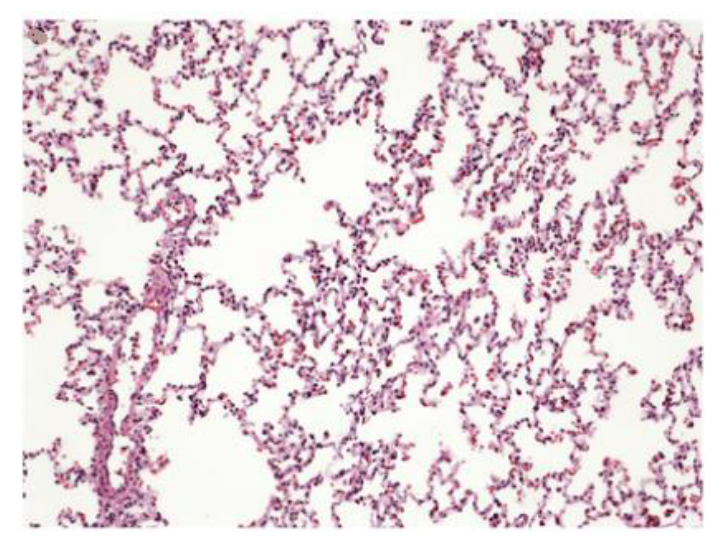

Figure 13b: Photomicrograph of H\&E stained histological section (magnification $10 \times 10$ ) showing the effect of vehicle PBS $\mathrm{pH} 7.4$ on the albino mice lung $24 \mathrm{~h}$ after intravenous injection $0.1 \mathrm{ml} / \mathrm{kg}$.

In Group 1 (Vehicle PBS pH 7.4, $0.1 \mathrm{ml} / \mathrm{kg}$ ) (Figure 13a and 13b) respectively, Group 3 (lomustine nanoparticles batch PD1, $0.1 \mathrm{ml}$ of 40 $\mathrm{mg} / \mathrm{kg}$ lomustine equivalent) (Figure 15a and 15b) respectively, Group 4 (lomustine nanoparticles batch PD4, $0.1 \mathrm{ml}$ of $40 \mathrm{mg} / \mathrm{kg}$ lomustine equivalent) and Group 5 (lomustine nanoparticles batch PD13, $0.1 \mathrm{ml}$ of $40 \mathrm{mg} / \mathrm{kg}$ lomustine equivalent) no microscopic change was observed in lungs and kidney. In Group 2 (Lomustine solution, $0.1 \mathrm{ml}$ of $40 \mathrm{mg} /$ 
Citation: Mehrotra A, Pandit JK (2015) Preparation and Characterization and Biodistribution Studies of Lomustine Loaded PLGA Nanoparticles by Interfacial Deposition Method. J Nanomed Nanotechnol 6: 328. doi:10.4172/2157-7439.1000328

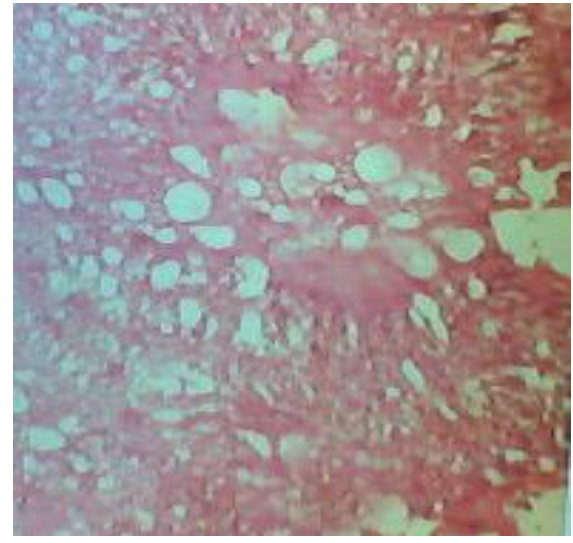

Figure 14a: Photomicrograph of H\&E stained histological section (magnification $10 \times 10$ ) showing the effect of lomustine on the albino mice kidney 24 $\mathrm{h}$ after intravenous injection of lomustine solution $0.1 \mathrm{ml}$ of $40 \mathrm{mg} / \mathrm{kg}$ lomustine equivalent.

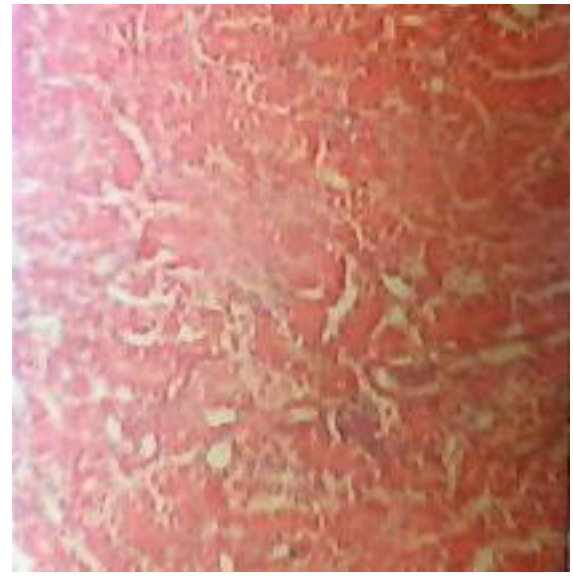

Figure 14b: Photomicrograph of H\&E stained histological section showing (magnification $10 \times 10$ ) the effect of lomustine on the albino mice lung $24 \mathrm{~h}$ after intravenous injection of lomustine solution 0.1 $\mathrm{ml}$ of $40 \mathrm{mg} / \mathrm{kg}$ lomustine equivalent.

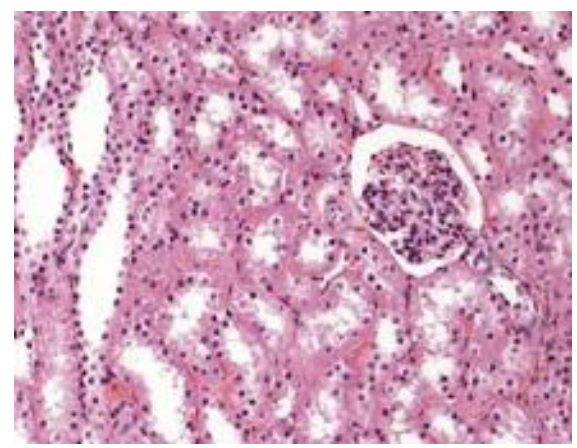

Figure 15a: Photomicrograph of H\&E stained histological section showing (magnification $10 \times 10$ ) the effect of lomustine on the albino mice kidney $24 \mathrm{~h}$ after intravenous injection of lomustine nanoparticles batch PD1, $0.1 \mathrm{ml}$ of $40 \mathrm{mg} / \mathrm{kg}$ lomustine equivalent.

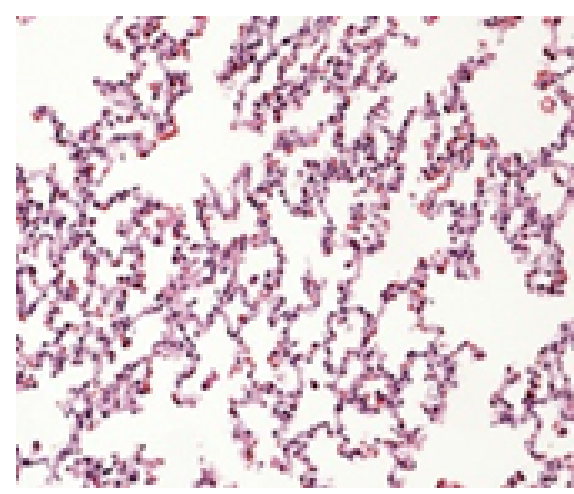

Figure 15b: Photomicrograph of H\&E stained histological section showing (magnification $10 \times 10$ ) the effect of lomustine on the albino mice lung $24 \mathrm{~h}$ after intravenous injection of lomustine nanoparticles batch PD1, $0.1 \mathrm{ml}$ of $40 \mathrm{mg} / \mathrm{kg}$ lomustine equivalent.

$\mathrm{kg}$ lomustine equivalent) the microscopic alterations were observed in lungs and kidney. Vacuoles in alveoli were seen in lungs (Figure 14a). Fatty changes were observed in kidney (Figure 14b).

\section{Conclusion}

It was concluded that formulation variables could be exploited in order to enhance the incorporation of lomustine into PLGA nanoparticles by interfacial deposition method. Based on the optimal parameters, it was found that lomustine-PLGA nanoparticles with expectable properties could be obtained. A technical advantage of the interfacial displacement method is that the formation of nanoparticles is not much influenced by the $\mathrm{L} / \mathrm{G}$ ratio and the polymer species. This method is simple, mild and practically easy because lomustineanticancer drug, demonstrated the highest encapsulation efficiency. Here it is notable that, mechanical strength and its ability to be formulated as a drug delivery device are controlled by molecular weight and its intrinsic viscosity. The intrinsic viscosity increase with the increase in PLGA concentration could increase the drug resistance diffusion into the aqueous phase and thus enhance the drug's incorporation into nanoparticles. Also, the in-vitro release behavior of lomustine from PLGA nanoparticles polymer matrix observed, showed that there was a fast initial release of drug during the first $24 \mathrm{hr}$, followed that the polymer chains undergo bulk degradation. The degradation generally occurs at a uniform rate throughout the PLGA matrix over $48 \mathrm{hr}$ in both in-vitro and in-vivo release with increased lung concentration of drug with slowed elimination and reduced toxicity.

\section{References}

1. Athanasiou KA, Niederauer GG, Agrawal CM (1996) Sterilization, toxicity biocompatibility and clinical applications of polylactic acid/polyglycolic acid copolymers. Biomaterials 17: 93-102.

2. Nagarwal RC, Kant S, Singh PN, Maiti P, Pandit JK (2009) Polymeric nanoparticulate system: a potential approach for ocular drug delivery. J Control Release 136: 2-13

3. Cheng FY, Wang SP, Su CH, Tsai TL, Wu PC, et al. (2008) Stabilizer-free poly(lactide-co-glycolide) nanoparticles for multimodal biomedical probes. Biomaterials 29: 2104-2112.

4. Pinto Reis C, Neufeld RJ, Ribeiro AJ, Veiga F (2006) Nanoencapsulation I. Methods for preparation of drug-loaded polymeric nanoparticles. Nanomedicine 2: 8-21.

5. Fessi H, Puisieux F, Devissaguet JPh, Ammoury N, Benita S (1989) Nanocapsule formation by interfacial polymer deposition following solvent 
Citation: Mehrotra A, Pandit JK (2015) Preparation and Characterization and Biodistribution Studies of Lomustine Loaded PLGA Nanoparticles by Interfacial Deposition Method. J Nanomed Nanotechnol 6: 328. doi:10.4172/2157-7439.1000328

displacement. Int J Pharm 55: R1-R4.

6. Holtzsherer VC, Benabbou S, Spenlehauer G, Veillard M, Couvreur P (1991) Methodology for the preparation of ultra-dispersed polymer systems, STP. Pharma Sci 1: 109-116

7. Fonseca C, Simões S, Gaspar R (2002) Paclitaxel-loaded PLGA nanoparticles: preparation, physicochemical characterization and in vitro anti-tumoral activity. J Control Release 83: 273-286.

8. Alleman E, Gurny R, Leroux JC (1998) Biodegradable nanoparticles of poly (lactic acid) and poly(lactic acid-co-glycolic acid) for parenteral administration. In: Lieberman HA, Rieger MM, Barker GS (eds.). Pharmaceutical Dosage Forms 3, Marcel Dekker, New York.

9. Kim IS, Lee SK, Park YM, Lee YB, Shin SC, et al. (2005) Physicochemical characterization of poly(L-lactic acid) and poly(D,L-lactide-co-glycolide) nanoparticles with polyethylenimine as gene delivery carrier. Int J Pharm 298: 255-262.

10. Mehrotra A, Pandit JK (2012) Critical process parameters evaluation of modified nanoprecipitation method on lomustinen nanoparticles and cytostatic activity study on L132 Human Cancer Cell Line. J Nanomed Nanotechol 3:1-8.

11. Al-Shammary FJ (1990) Lomustine, Comprehensive description: Analytical Profiles Drug Substances. In: Florey K (eds.), Academic Press Inc, London.

12. Jiang XY, Zhou CS, Tang KW (2003) Preparation of PLA and PLGA nanoparticles by binary organic solvent diffusion method. J Cent South Univ Technol 10: 202-206

13. Song X, Zhao Y, Hou S, Xu F, Zhao R, et al. (2008) Dual agents loaded PLGA nanoparticles: systematic study of particle size and drug entrapment efficiency. Eur J Pharm Biopharm 69: 445-453.

14. Cegnar M, Kos J, Kristl J (2004) Cystatin incorporated in poly(lactide-coglycolide) nanoparticles: development and fundamental studies on preservation of its activity. Eur J Pharm Sci 22: 357-364.

15. Nafee N, Taetz S, Schneider M, Schaefer UF, Lehr CM (2007) Chitosancoated PLGA nanoparticles for DNA/RNA delivery: effect of the formulation parameters on complexation and transfection of antisense oligonucleotides. Nanomedicine 3: 173-183.

16. Barnes TJ, Prestidge CA (2000) PEO-PPO-PEO block copolymers at the emulsion droplet-water interface. Langmuir 16: 4116-4121.

17. Redhead HM, Davis SS, Illum L (2001) Drug delivery in poly(lactide-coglycolide) nanoparticles surface modified with poloxamer 407 and poloxamine
908. in vitro characterisation and in vivo evaluation. J Control Release 70:353 363.

18. Zou W, Liu C, Chen Z, Zhang N (2009) Studies on bioadhesive PLGA nanoparticles: A promising gene delivery system for efficient gene therapy to lung cancer. Int J Pharm 370: 187-195.

19. Shaikh J, Ankola DD, Beniwal V, Singh D, Ravi Kumar MNV (2009) Nanoparticle encapsulation improves oral bioavailability of curcumin by at least 9-fold when compared to curcumin administered with piperine as absorption enhancer. Eur J Pharm Sci 37: 223-230.

20. Wang XQ, Dai JD, Chen Z, Zhang T, Xia GM, et al. (2004) Bioavailability and pharmacokinetics of cyclosporine A-loaded $\mathrm{pH}$-sensitive nanoparticles for oral administration. J Control Release 97: 421-429.

21. Danhier F, Lecouturier N, Vroman B, Jerome C, Marchand BJ, et al. (2009) Paclitaxel-loaded PEGylated PLGA-based nanoparticles: In vitro and in vivo evaluation. J Control Release 133: 11-17.

22. Song X, Zhao Y, Wu W, Bi Y, Cai Z, et al. (2008) PLGA nanoparticles simultaneously loaded with vincristine sulfate and verapamil hydrochloride: Systematic study of particle size and drug entrapment efficiency. Int J Pharm 350: $320-329$.

23. Muthu MS, Rawat MK, Mishra A, Singh S (2009) PLGA nanoparticle formulations of risperidone: preparation and neuropharmacological evaluation. Nanomedicine 5: 323-333.

24. Corrigan OI, Li X (2009) Quantifying drug release from PLGA nanoparticulates Eur J Pharm Sci 37: 477-485.

25. Blasi P, Schoubben A, Giovagnoli S, Perioli L, Ricci M, et al. (2007) Ketoprofen poly(lactide-co-glycolide) physical interaction. AAPS PharmSciTech 8: Article 37.

26. Brigger I, Dubernet C, Couvreur P (2002) Nanoparticles in cancer therapy and diagnosis. Adv Drug Deliv Rev 54: 631-651.

27. Seal BL, Otero TC, Panitch A (2001) Polymeric biomaterials for tissue and organ regeneration. Mater Sci Eng R 34: 147-230.

28. Lherm C, Mqler RH, Puisieux F, Couvreur P (1992) Alkylcyanoacrylate drug carriers: II. Cytotoxicity of cyanoacrylate nanoparticles with different alkyl chain length. Int J Pharm 84: 13-22.

29. Lomustine Comprehensive Description: Analytical Profiles Drug Substances. In: Florey K (eds.), Academic Press Inc, NewYork. 